\title{
Viewpoint
}

\section{Do diets rich in polyunsaturated fatty acids affect disease activity in rheumatoid arthritis?}

\author{
L GAIL DARLINGTON \\ From the Epsom District Hospital, Epsom, Surrey
}

Key words: cod liver oil, evening primrose oil, dietary supplements, prostaglandins, leucotrienes.

Patients have long believed that fish oils in general, and cod liver oil in particular, may help to relieve the symptoms of rheumatoid arthritis. More recently it has been suggested that evening primrose oil may also be beneficial for arthritic patients.

What then is the scientific evidence to support these claims and is it of sufficient promise to warrant further, orthodox investigation?

\section{Diets supplemented with fish oil}

Fish oils contain long chain, highly polyunsaturated, omega-3 fatty acids, particularly eicosapentaenoic (20:5 omega-3) and docosahexaenoic (22:6 omega3 ) acid of the linolenic or omega- 3 class, unlike linoleic acid (18:2 omega-6) which is the predominant polyunsaturated fatty acid in a Western diet. Oxidative metabolism of arachidonic acid, eicosapentaenoic acid, and docosahexaenoic acid by the 5 -lipoxygenase pathway is shown in Fig. 1. Mast cell activation and the arachidonic acid metabolic pathways are shown in Fig. 2.

One theory behind fish oil therapy is that fatty acids, such as $\alpha$-linolenic acid from fish oils, are converted into prostaglandins of the third series, whereas the saturated fats of commonly consumed Western diets, e.g., linoleic acid, are converted via arachidonic acid into prostaglandins of the second series. Because prostaglandins of the second series are actively involved in the rheumatoid process there may well be some benefit for a patient in diverting prostaglandin production from the inflammatory second series into the less inflammatory

Accepted for publication 15 June 1987.

Correspondence to Dr L Gail Darlington. Epsom District Hospital, Dorking Road. Epsom, Surrey KT19 7EG. third series with, perhaps, a reduction in rheumatoid activity. The pro-inflammatory effects of the 2 series prostaglandins and the 4 series leucotrienes are shown in Fig. 3.

In 1985 Lee et al concluded that diets enriched with fish oil may have anti-inflammatory effects by inhibiting the 5-lipoxygenase pathway in neutrophils and monocytes and by inhibiting the leucotriene B4 mediated functions of neutrophils. ${ }^{1}$

In fact, as Meade and Mertin pointed out in 1978, a wide variety of fatty acids produce effects on the immune system. ${ }^{2}$ There may, therefore, be a variety of additional ways in which dietary supplementation with oils may affect rheumatoid activity, but the changes in prostaglandin production described above form the basis of current hypotheses.

Few detailed studies have yet been published on the use of fish oils alone in rheumatoid arthritis in humans, though Kremer et al in 1985 described improvement in patients with rheumatoid arthritis using a diet that restricted saturated fat and was high in polyunsaturated fats, with an additional supplement of eicosapentaenoic acid, i.e., the 'Eskimo diet'. ${ }^{3}$

In 1986 McColl et al reported that dietary supplementation with omega-3 fatty acid in the form of eicosapentaenoic acid reduced the severity of adjuvant induced inflammation in rats. ${ }^{4}$

Also in 1986 Sperling et al investigated the effects of dietary fish oils on leucocyte function in vitro, leucotriene generation, and disease activity in patients with rheumatoid arthritis on stable, nonsteroidal anti-inflammatory regimens. ${ }^{5}$ Sperling felt that the results seemed to indicate a synergistic effect with fish oil and non-steroidal anti-inflammatory drugs and concluded that the addition of dietary fish oil to non-steroidal anti-inflammatory 


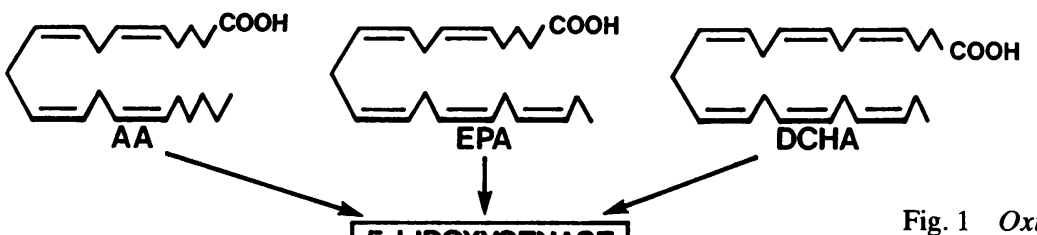

Fig. 1 Oxidative metabolism of arachidonic $\frac{}{\mathrm{D}}$ 5 LIPOXYGENASE acid $(A A)$, eicosapentaenoic acid (EPA), and docosahexaenoic acid (DCHA) by the 5-lipoxygenase pathway.
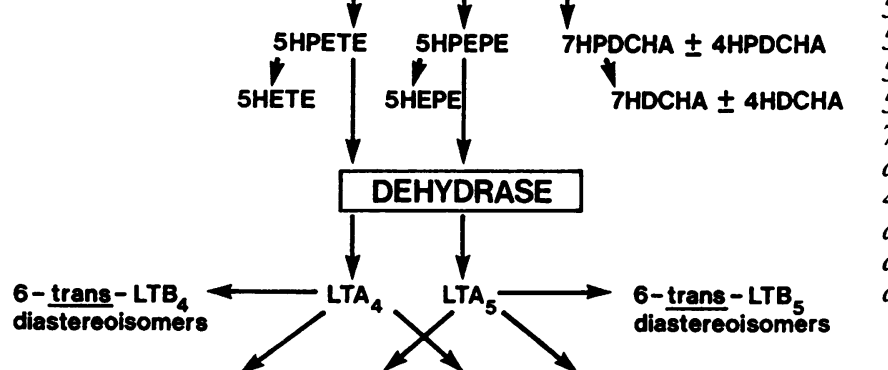

6-trans - LTB $_{5}$ diastereoisomers 5HPETE $=5$-hydroperoxyeicosatetraenoic acid $\overrightarrow{0}$ $5 H E T E=5$-hydroxyeicosatetraenoic acid; 5HPEPE =5-hydroperoxyeicosapentaenoic acid 7HPDCHA =7-hydroperoxydocosahexaenoic acid; 4HPDCHA=4-hydroperoxydocosahexaenoic $A$ acid; $7 H D C H A=7-h y d r o x y d o c o s a h e x a e n o i c$. acid; $4 H D C H A=4$-hydroxydocosahexaenoic acid; and $L T=$ leucotriene.

EPOXIDE HYDROLASE GLUTATHIONE-S-TRANSFERASE
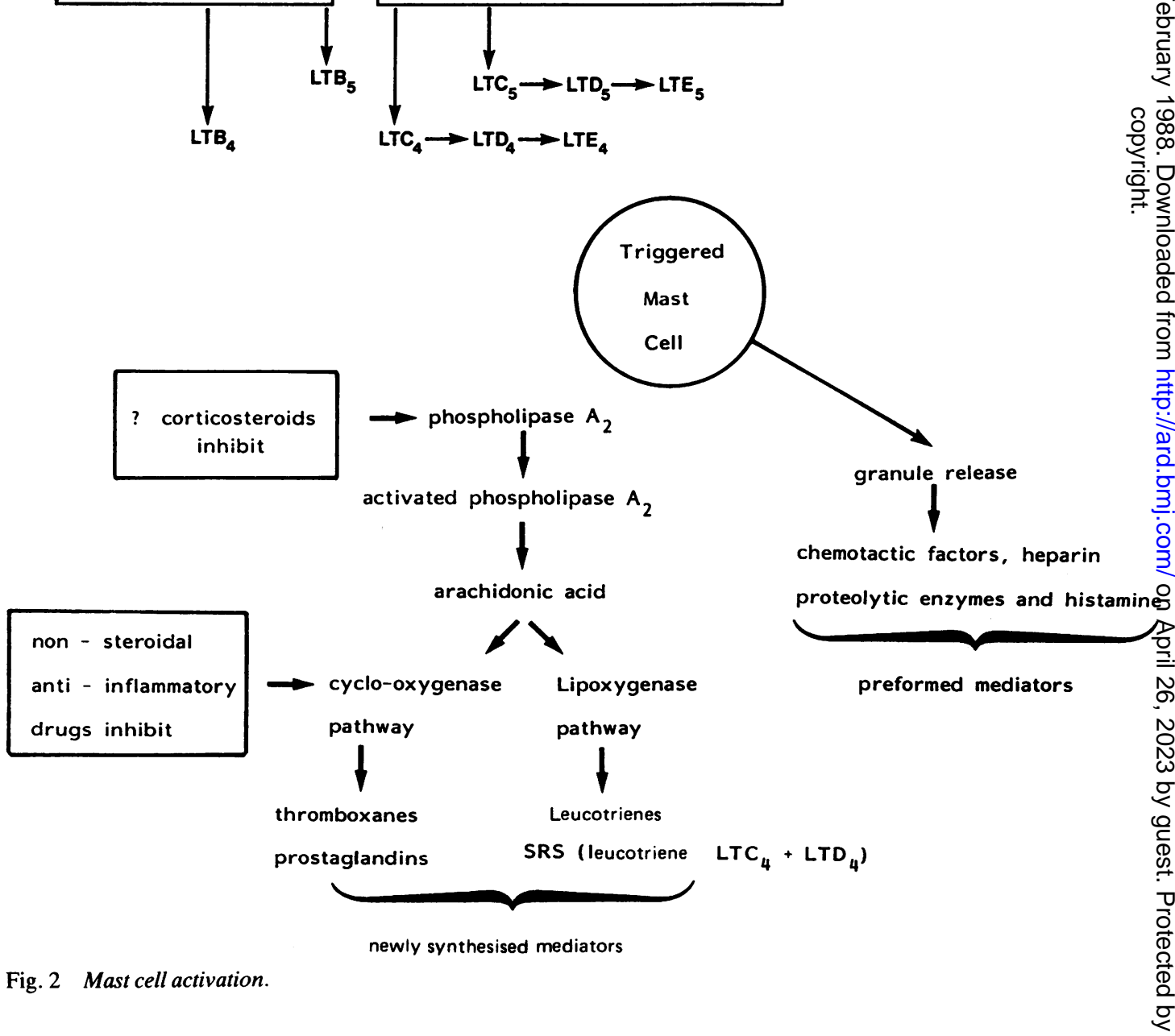
2) Inflammatory activators Avascular permeability microthrombi : via PAF (platelet activating factor)

3) Spasmogenic agents

$\left.\begin{array}{l}\text { - bronchial smooth muscle } \\ \text { contraction } \\ \text { - mucosal oedema } \\ \text { - mucus secretion }\end{array}\right\} \mathrm{LTC}_{4}$

Fig. 3 Pro-inflammatory effects of the 2 series prostaglandins $(P G)$ and the 4 series leucotrienes $(L T)$.

4)

$$
\begin{aligned}
& \text { Produce } \\
& \text { - heat } \\
& \text { - redness } \\
& \text { - pain } \\
& \text { - oedema }
\end{aligned}
$$

\section{with histamine and kinins}

therapy for six weeks significantly improved clinical and biological indices.

Our own controlled study of the use of fish oils in rheumatoid patients is nearing completion and doubtless other such studies are in progress, but the exact role, if any, of fish oils in the treatment of rheumatoid arthritis has yet to be defined.

Nonetheless, it is reassuring to remember that fish oils may be considered as foods rather than drugs and they certainly appear to be extremely safe.

Moreover, dietary fish oils have been shown to reduce both plasma cholesterol and triglyceride in normal $^{6}$ and hyperlipidaemic subjects. ${ }^{\top}$ In 1985 Singer et al showed that the type of fish eaten was also important as a mackerel diet appeared to have a significant effect in lowering serum lipids and blood pressure whereas a herring diet did not. ${ }^{8}$

Furthermore, fish oils may have a cardioprotective role as it has been reported that the consumption of only $30 \mathrm{~g}$ of fish a day is associated with a more than $50 \%$ reduction in mortality from coronary artery disease. ${ }^{9}$ It seems, therefore, that fish oils may not only help the joints of patients with rheumatoid arthritis but may protect their coronary arteries also.

\section{Diets supplemented with evening primrose oil}

It is believed that treatment with $\gamma$-linolenic acid, from evening primrose oil, may produce a fall in production of prostaglandins of the second series similar to that which is thought to be induced by fish oils. When evening primrose oil is used, however, there is increased production of prostaglandins of the first series (e.g. $\mathrm{PGE}_{1}$ ), which have anti-inflammatory activity.

In 1982 Kunkel et al examined in rats the effect of evening primrose oil and its ability to modulate inflammatory cells and to suppress chronic inflammation. ${ }^{10}$ The authors showed that the chemotactic activity of rat polymorphonuclear leucocytes to a synthetic chemoattractant was significantly impaired and, in addition, chronic proliferative adjuvant arthritis was greatly suppressed in those rats whose diets were supplemented with the oil.

In contrast, however, in 1983 Hansen et al treated 20 patients with active rheumatoid arthritis using evening primrose oil for 12 weeks but found no effect from the treatment on any of the subjective and objective variables measured. ${ }^{11}$

In 1986, in a most interesting study, Belch et al investigated the effect both of evening primrose oil alone and of an evening primrose oil/fish oil combination in patients with rheumatoid arthritis. ${ }^{12}$ They concluded that both evening primrose oil alone and also evening primrose oil with fish oil produced significant clinical improvement and allowed some patients significantly to reduce or 
even to stop non-steroidal anti-inflammatory drugs. They found no evidence, however, to suggest that the oils acted as disease modifying agents.

The data so far on evening primrose oll look very interesting but more careful work will be required to give further information on safety and compliance.

It is likely that the cure for rheumatoid arthritis will eventually come as a result of careful research into the immunology of the disease. It is also probable, however, that immunological progress will need time to be transformed into clinical reality and, in the meantime, all forms of dietary manipulation used by patients, including supplementation of the diet with oils, should be investigated as scientifically as any other form of treatment.

Doctors have been wary of encouraging patients in their wish to take oils, in the same way that they have been wary about other forms of dietary manipulation therapy. This is understandable as, until recently, there has been little scientific evidence to support patients' enthusiasm.

Now, however, the situation appears to be changing and careful scientific studies are being undertaken in the United Kingdom and also abroad in an attempt to determine the true facts. Only thus will orthodox clinicians be able to separate the sense from the nonsense and obtain the information with which they can advise their patients with confidence based on scientific findings.

\section{References}

1 Lec T H, Hoover R L, Williams J D, et al. Effect of dictary enrichment with cicosapentaenoic and docosahexaenoic acios on in vitro neutrophil and monocyte leukotriene generation are? neutrophil function. $N$ Engl $J$ Med 1985; 312: 1217-24.

2 Meade C J, Mertin J. Fatty acids and immunity. Adv Lip $\overline{\bar{x}}$ Res 1978; 16: 127-65.

3 Kremer J M, Michalck A V, Lininger L, et al. Effects of manipulation of dietary fatty acids on clinical manifestations rheumatoid arthritis. Lancet 1985 ; i: 184-7.

4 McColl S R. Whitchouse M W, Cleland L G, Hurst N D Dietary supplementation with omega-3 fatty acids reducs severity of adjuvant-induced arthritis in rats [Abstract]. Bred Rheumatol 1986; 25: 106.

5 Sperling R, et al. Paper presented at the 50th annual meeting of the American Rheumatism Association, 3-7 June 1986. Ncy Orleans. Advances in Rheumatology 1986; $1: 8$.

6 Harris W S, Connor W E, McMurry M P. The comparatiog reductions of the plasma lipids and lipoproteins by dieta polyunsaturated fats: salmon oil vs. vegetable oils. Metabolisin 1983: 32: 179-84.

7 Phillipson B E, Rothrock D W, Connor W E, Harris W Su Illingworth D R. Reduction of plasma lipids, lipoproteins a apoproteins by dictary fish oils in patients with hypertriglyce $\hat{C}^{\circ}$ daemia. $N$ Engl J Med 1985; 312: 1210-6.

8 Singer $\mathrm{P}$, Wirth $\mathrm{M}$, Voigt $\mathrm{S}$, et al. Blood pressure- and lipidlowering effect of mackerel and herring diet in patients with mild essential hypertension. Atherosclerosis 1985; 56: 223-30.

9 Kromhout D, Bosschicter E B. Coulander C de L. The inver relation between fish consumption and 20-year mortality frots coronary heart discasc. $N$ Engl J Med 1985; 312: 1205-9

10 Kunkel S L, Ogawa H, Ward P A, Zuricr R B. Suppression of chronic inflammation by evening primrose oil. Prog LipifR 1982; 20: 885-8.

11 Hansen T M, Lerche A, Kassis V, Lorenzen I, Sondergaamd] Treatment of rheumatoid arthritis with prostaglandin $E_{1}$ prescomsors cis-linoleic acid and $\gamma$ linolenic acid. Scand $J$ RheuFta 1983; 12: 85-8.

12 Belch J J F, Ansell D. Madhok R, Sturrock R D. The effects @of altering dietary essential fatty acids on requirements for no steroidal anti-inflammatory drugs in patients with rhcumato@ arthritis: a double blind placebo controlled study [Abstract]. Fi $J$ Rheumatol 1986; 25 (suppl): 75. 